\title{
Diagnóstico del clima organizacional desde la perspectiva del docente en la Facultad de Ciencias Agrotecnológicas
}

Diagnosis of the organizational climate from the perspective of the teacher in the Faculty of Agrotechnological Sciencies

\begin{abstract}
Tarín Estrada Lorena A. ${ }^{1}$, Ortega Rodríguez Anabel ${ }^{1 凶}$, García Muñoz Silvia A. ${ }^{1}$, Leyva Chávez Arwell N. ${ }^{1}$, Casillas García Jesús U. ${ }^{1}$, Lujan Aguirre Ramón S. ${ }^{1}$

${ }^{1}$ Facultad de Ciencias Agrotecnológicas, Universidad Autónoma de Chihuahua Campus I, Chihuahua, Chih., México. Tel. (614)4391844.

${ }^{凶}$ Autor de correspondencia: aortegar@uach.mx.
\end{abstract}

Recibido: $12 / 03 / 2018$
Aceptado: $13 / 05 / 2018$

\section{RESUMEN}

La investigación tuvo como objetivo evaluar el clima organizacional en la Facultad de Ciencias Agrotecnológicas de la Universidad Autónoma de Chihuahua y a partir de éste se determina el nombre del proyecto: Diagnóstico del clima organizacional desde la perspectiva de los docentes de la Facultad de Ciencias Agrotecnológicas. Este trabajo pretende refrescar un estudio investigativo sobre el clima organizacional que se realizó anteriormente en la Facultad. La combinación de experiencia, práctica, ideas y conocimientos teóricos brindan el soporte necesario para construir y proponer procesos de intervención que establezcan un panorama real y un mejoramiento continuo al clima organizacional encontrado en la Facultad. La investigación es de tipo descriptivo cuantitativo en la modalidad de campo. El total de encuestas realizadas fue de 32 .

Palabras clave: Clima organizacional, universidad, FACIATEC, calidad, competitividad.

\begin{abstract}
The objective of this research was to evaluate the organizational climate in the Faculty of Agrotechnology Sciences at the Autonomous University of Chihuahua and from this is determined the name of the Project: Diagnostics of the organizational climate from the perspective of the teachers of the Faculty of Agrotechnology Sciences. This work aims to refresh a study research on the organizational climate that was previously on the faculty. The combination of experience, practice, ideas and knowledge acquired provide the necessary support to build and propose intervention processes to establish a real picture and a continuous improvement to the organizational climate found in the faculty. The Research is quantitative descriptive in the field modality. The total number of surveys was 32 .
\end{abstract}

Key Words: Organizational Climate, University, FACIATEC, Quality, Competitiveness. 


\section{INTRODUCCIÓN}

En la actualidad, las instituciones se ven enfrentadas al fenómeno de la globalización, lo cual, les exige ser más competitivas. Para poder lograr esto, se debe considerar a los recursos humanos como los activos más importantes, en este caso, el personal académico o docentes, ya que la habilidad de las instituciones y/o organizaciones para alcanzar sus objetivos está determinada por la productividad individual y colectiva.

El clima organizacional es el factor que impulsa al éxito en las instituciones, entonces surge la inquietud de sembrar la reflexión sobre la importancia del mismo como una herramienta estratégica que debe ser considerada por la

Facultad para alcanzar altos grados de productividad.

Es por eso la importancia del presente estudio ya que todo esto permitirá y desarrollara climas de trabajo que permitirán la pro actividad de los docentes.

Por cuestiones de tiempo en la realización de dicha investigación, este estudio solo contempló a un porcentaje del personal docente, profesores de tiempo completo, profesores de medio tiempo y profesores de hora clase de los cinco programas educativos que conforman la Facultad de Ciencias Agrotecnológicas de la Universidad Autónoma de Chihuahua.

Se consideró pertinente estudiar el clima organizacional y la satisfacción laboral, pretendiendo aportar los elementos necesarios para la mejora de dichos procesos y los que estos puedan afectar.

\section{MATERIALES Y MÉTODOS}

Dicha investigación se efectuó con 32 docentes de la Facultad de Ciencias Agrotecnológicas de la Universidad Autónoma de Chihuahua, entre los cuales hay docentes de tiempo completo y hora clase, este número de personas no coincidirá con el número real de docentes que trabajan en dicha Facultad, ya que solo el $33.33 \%$ fueron los que contestaron el cuestionario que se les aplico, la otra parte por diversos motivos no les fue posible contestarlo. Esta investigación pretende estudiar la relación existente entre el clima organizacional, el comportamiento y el desempeño del docente en dicha Facultad.

Los 32 docentes encuestados son especificados de esta forma:

- Profesores de tiempo completo: 11

- Profesores de hora clase: 21

Entre los cuales cuentan con los siguientes grados de estudio:

- Licenciatura: 5

- Maestría: 23

- Doctorado: 4

De los 32 docentes seis pertenecen al Programa de Mejoramiento del Profesorado (PROMEP); y solo uno pertenece al Sistema Nacional de Investigadores (SNI).

Se aplicó un cuestionario el cual consiste de 58 preguntas presentadas en forma de afirmaciones o juicios ante los cuales se pide la reacción de los sujetos. Se presenta cada afirmación y se pide al sujeto que externe su reacción eligiendo uno de los cinco puntos de la escala tipo Likert: Totalmente de acuerdo, De acuerdo, Neutral, En desacuerdo y Totalmente en desacuerdo. Las afirmaciones tienen dirección favorable $\mathrm{o}$ positiva y desfavorable o negativa, siendo esto muy importante para codificar las alternativas de respuesta. Una afirmación positiva significa que el sujeto califica favorablemente el objeto de actitud, y cuanto más de acuerdo con la afirmación, la actitud será más favorable. Las preguntas se diseñaron de una manera simple y comprensible y para aumentar la realidad y 
calidad de la información, el cuestionario será auto administrado y anónimo.

\section{RESULTADOS}

La gran mayoría de los docentes indican estar satisfechos con el nivel académico de la Facultad, consideran que son buenos modelos para sus estudiantes y que los preparan eficientemente para el momento de su egreso, a su vez los impulsan al intercambio estudiantil; también reflejan que las tutorías que les son asignadas han generado eficazmente cambios en los alumnos ya que han mejorado su nivel académico.

En cuanto a las actividades académicas, los resultados son que están contentos y de acuerdo con las clases que imparten, y con su trabajo como docentes, así como las investigaciones en las que participan, y aunque para una gran parte de los docentes las investigaciones no aplican para ellos, la mayoría indica estar satisfecho con sus investigaciones.

Pero, así como están satisfechos en muchas cosas, también se tienen resultados donde la mayoría están insatisfechos en otras, una de ellas es que consideran que no participan constantemente en programas de movilidad académico, donde ellos se pudieran desarrollar profesionalmente.

En cuanto a comunicación, la mayoría de los docentes conocen los estatutos, reglamentos y planes de estudio de la UACH, pero también gran parte de ellos no lo conocen, ya sea por falta de interés por parte de ellos o, por parte de la universidad que no les ha hecho llegar dicha información; referente al PRODES y al PIFI, la gran mayoría contesto que no aplican a dichos programas.

Más del 50\% de los docentes están satisfechos con la información oportuna que se les brinda en cuanto eventos de capacitación; referente a convocatorias para proyectos de investigación la mayoría se siente indiferente con esto y no le toman tanta importancia, por lo que respecto a comunicación general en la Facultad consideran que esta fluye correctamente, y aunque un gran porcentaje de ellos no lo ven así, la mayoría se siente satisfecho con la información y comunicación con la que cuentan en FACIATEC.

Sobre aspectos de administración, los resultados arrojan que la mayoría de los docentes se sienten indiferentes en cuanto a la claridad que existe de la distribución de los recursos financieros, así como la eficiencia de los trámites que hay para asistir a eventos académicos, a programas de capacitación y desarrollo profesional y al cumplimiento de los requerimientos de contratación de personal, por lo que gran parte de la docencia de FACIATEC respondió neutral, lo que quiere decir que no están satisfechos ni insatisfechos con ello. Mas sin embargo se sienten satisfechos y de acuerdo con la administración de la Facultad.

Respecto a toma de decisiones, los docentes se sienten tomados en cuenta para la elaboración de diversos programas, también consideran que sus opiniones son tomadas en cuenta en las sesiones del consejo técnico, así como en decisiones asociadas a su trabajo de docentes, pero por el contrario consideran que no tienen una vida colegiada significativa, dejando como resultado que es mayor los resultados positivos que se tienen que los negativos.

Sobre el ambiente laboral, se tienen resultados mayormente positivos, donde consideran que su unidad académica se preocupa por impulsar actividades de vinculación, y de investigación, así como de la formación de sus estudiantes; también contestaron estar de acuerdo en cuanto al reconocimiento laboral que la Facultad les brinda a sus académicos, al positivismo en su estado de ánimo y al ambiente en general en que se desempeñan, de igual manera consideran estar satisfechos con el acuerdo de transparencia, donde se refleja que ha mejorado la confianza y 
comunicación en la institución; pero por otro lado reflejan que la UACH no considera a las personas como lo más importante, ya que para esto la mayoría respondió estar en desacuerdo.

La mayor parte de la docencia de FACIATEC indica estar de acuerdo a los apoyos que la institución les brinda para su desempeño, a sus responsabilidades de docencia, investigación y gestión, en general consideran que las condiciones de trabajo de la institución son las adecuadas, con lo único que están un tanto en desacuerdo es los apoyos para realizar estudios de posgrado, lo cual consideran que necesitan se les apoye más en esto.

Respecto a las certificaciones y acreditaciones con las que cuentan la Facultad, la mayor parte de los docentes indican estar de acuerdo en que gracias a ellas se ha mejorado la calidad del servicio, FACIATEC se ha vuelto más competitiva y tienen programas educativos de calidad, también la mayoría conoce si la carrera que imparte está acreditada lo cual es muy positivo porque reflejan la excelencia de sus clases.

En general los docentes de FACIATEC se sienten satisfechos en pertenecer a la $\mathrm{UACH}$, contentos con su comunidad y con los logros que se han alcanzado. A pesar de algunas insatisfacciones, el mayor porcentaje indica satisfacción en su entorno laboral.

\section{CONCLUSIONES}

El conocimiento del clima organizacional proporciona retroalimentación acerca de los procesos que determinan los comportamientos organizacionales, permitiendo, además, introducir cambios planificados tanto en las actitudes y conductas de los miembros, como en la estructura organizacional. La importancia de esta información se basa en la comprobación de que el clima organizacional influye en el comportamiento manifiesto de los miembros, a través de las percepciones estabilizadas que filtran la realidad y condicionan los niveles de motivación laboral y rendimiento profesional entre otros. Para que los docentes lleguen a manifestar claramente sus necesidades de autorrealización y querer desarrollarlas en beneficio de la institución a la cual prestan su servicio, deben estar no solo satisfechas, sino motivadas en y con su trabajo. Así, tanto el ambiente de trabajo como el trabajo en sí, son factores importantes y básicos para el desarrollo del potencial humano.

\section{LITERATURA CITADA}

Aguera Ibañez Enrique. 2004. Liderazgo y compromiso social. $1^{\mathrm{a}}$ Edición. Puebla. Puebla.

Castañeda Luis. 2004. Un plan de formación en liderazgo para jóvenes. $1^{a}$ Edición. México.

Chiang Margarita, Martín Ma. José, Núñez Antonio. 2010. Relación entre clima organizacional y satisfacción laboral. $1^{\mathrm{a}}$ Edición. Madrid.

Chiavenato Idalberto. 2009. Comportamiento Organizacional. Dinámica del éxito en las organizaciones. $2^{\text {a }}$ Edición. México. D. F.

Diez Freijeiro Sara. 2006. Técnicas de comunicación. La comunicación en la empresa. $1^{a}$ Edición. España.

Dr. Cole Donald W., Butterfield Gaynor Eric. 2005. Desarrollo organizacional y desarrollo ejecutivo. $1^{\mathrm{a}}$ Edición. Buenos Aires. Argentina.

Ezequiel Ander-Egg y Aguilar Martin José. 2001. El trabajo en equipo. $1^{\mathrm{a}}$ Edición. México. D.F.

Koenes Avelina. 1998. Gestión eficaz del trabajo en equipo. $1^{\text {a }}$ Edición. Madrid. España.

Martínez Guillen María del Carmen. 2003. La gestión empresarial. Equilibrando objetivos y valores. $1^{\text {a }}$ Edición. España. 
Puchol Luis. 2007. Dirección y gestión de recursos humanos. $7^{\mathrm{a}}$ Edición. España.

Stephen P. Robbins. 1998. Fundamentos de comportamiento organizacional. $5^{\text {a }}$ Edición, Naucalpan de Juárez. Estado de México.

Stephen P. Robbins. 2004. Comportamiento

Organizacional. $10^{a}$ edición. México.

Stephen P Robbins, Timothy A. Judge 2009.

Comportamiento Organizacional. $13^{\mathrm{a}}$

Edición. México.

Tejeda F (coordinador)., Giménez M (coordinador)., Viladot V., Gan B., Fandos B., Jiménez G., y González S. 2007. Formación de formadores. $1^{\mathrm{a}}$ Edición. Madrid. España.

Velázquez Ramírez Diana. 2012. Clima

Organizacional en la Universidad del Valle.

Documento de trabajo diagnóstico. Cali. Colombia.

Zuazua Iriondo Alberto. 2007. El proyecto de autorrealización. Cambio, curación, y desarrollo.

\section{Obra de consulta en línea}

Administración II, ingeniería en transporte. Tema 4.4 componentes y resultados del clima organizacional. 2009. http://administracion2transporte.blogspot.m x/2009/02/componentes-y-resultados-delclima.html

Arias G. Ma. de Lourdes, Arias G. Enrique, Arias G. Jesús. (2013). LA educación superior en México: financiamiento de instituciones y estudiantes. Tecsistecatl. Volumen5.

http://www.eumed.net/rev/tecsistecatl/n14/e ducacion.html

Informador.mx. 2015. Matrícula de educación superior es de 3.8 millones: SEP http://www.informador.com.mx/mexico/20 15/604452/6/matricula-deeducacion superior-es-de-38-millones-sep.htm
Innova block IIC. Motivación y reconocimiento. http://blog.iic.uam.es/2013/04/motivaciony-reconocimiento/

Martínez Coll Juan Carlos. Actualizaciones para el management y el desarrollo organizacional.

https://books.google.com.mx/books?id=Dr RHb9Lp84C\&printsec $=$ frontcover\#v=onepa ge\&q\&f=false.

Narro Robles, José; Martuscelli Quintana, Jaime y Barzana García, Eduardo (Coord.) (2012) Plan de diez años para desarrollar el Sistema Educativo Nacional. [En línea]. México: Dirección General de Publicaciones y Fomento Editorial, UNAM. http://www.planeducativonacional.unam.m $\mathrm{X}$

Serzo Hery. Clásicos de la gerencia. 1984 http://reddinconsultants.com/espanol/wpcontent/uploads/2012/12/Rensis-Likert-yMcGregor.pdf.

Valencia O. Alicia, Toledo G. José y Hernández A. Hermilio. Diagnóstico de clima organizacional: caso del ingenio El Molino. http://www.eumed.net/librosgratis/2013a/1333/climaorganizacional.html

\section{Tesis doctoral, trabajo académico en línea}

Brito Yves, Juan Carlos Jiménez. 2009. Evaluación del Clima Organizacional Universitario. Caso: Facultad de Ingeniería - Universidad de Carabobo. Venezuela. http://2fwww.redalyc.org/articulo.oa?id=21 5016874006.

Duran Claudia, Atlante María Estela, Giordano Diana. 2010. El Clima Organizacional en la Universidad: Modelo de Medición para el Personal de Apoyo Universitario. América del

Sur. https://repositorio.ufsc.br/bitstream/handle/ 123456789/96677/DURAN.pdf? sequence $=$ 1. 
Lourdes Penoni, Montserrat Cosp. 2007. $\mathrm{http} / / / \mathrm{www}$.adecomunicaciones.com/wpMemoria de la licenciatura: La motivación content/plugins/downloadsorganización Guyra Paraguay manager/upload/cosp-penoni.pdf 